\title{
ON THE LOCAL BEHAVIOR OF CERTAIN HOMEOMORPHISMS
}

\author{
By Melkana Brakalova and James a. Jenkins
}

1. Let $w(z)$ be an ACL homeomorphism of the unit disc $U_{0}$ into itself such that $w(0)=0$. At regular points $z(=x+i y)$ we define

$$
w_{z}=\frac{1}{2}\left(w_{x}-i w_{y}\right), \quad w_{\bar{z}}=\frac{1}{2}\left(w_{x}+i w_{y}\right)
$$

and the complex dilatation

$$
\kappa(z)=w_{\bar{z}} / w_{z} .
$$

Later we will require further that the directional derivatives of $w(z)$ satisfy certain integrability conditions (Condition 3.1).

It is well known that if $\|\kappa\|_{\infty} \leqq q<1$ then $w(z)$ is $K$-quasiconformal ( $K$ q. c.) with $K=(1+q) /(1-q)$. As usual we say that $w(z)$ is conformal at $z=0$ if

$$
\lim _{z \rightarrow 0}(w(z) / z)=C, \quad C \neq 0 .
$$

If $|w(z)| \sim A|z|$ as $z \rightarrow 0, A>0$ we say that $w(z)$ is asymptotically a rotation on circles as $z \rightarrow 0$ if for an appropriate choice of the arguments

$$
\arg w\left(r e^{i \theta_{2}}\right)-\arg w\left(r e^{i \theta_{1}}\right)-\left(\theta_{2}-\theta_{1}\right)
$$

tends to zero uniformly in $\theta_{1}$ and $\theta_{2}$ as $r$ tends to zero.

Our objective is to study the behavior of $w(z)$ as $z$ tends to zero allowing the possibility that $\|\kappa\|_{\infty}=1$. Our main results are contained in Theorem 1.1 and Theorem 1.2 .

THEOREM 1.1. If $\phi=\arg z$,

$$
\iint_{U_{0}} \frac{|\kappa|^{2}+\left|\mathscr{R} e^{-2 \imath \phi} \kappa\right|}{1-|\kappa|^{2}} \frac{d A}{|z|^{2}}<\infty
$$

then

$$
|w(z)| \sim A|z|, \quad z \rightarrow 0, \quad A>0
$$

and $w(z)$ is asymptotically a rotation on circles, i.e.,

Received August 23, 1993. 


$$
\arg w\left(r e^{i \theta_{2}}\right)-\arg w\left(r e^{i \theta_{1}}\right)-\left(\theta_{2}-\theta_{1}\right)
$$

tends to zero uniformly in $\theta_{1}$ and $\theta_{2}$ as $r$ tends to 0 .

The fact that (1.1) implies (1.2) was proved in [6] for $w(z) K$ q.c. Here we do not assume $K$-quasiconformality and instead of using Teichmüller's Modulsatz we use the technique given by [3; Lemma 3]. This makes possible the proof that the mapping is asymptotically a rotation, a new result which provides a significant step in the study of conformality at $z=0$, as evidenced in the following statement.

COROLlaRY 1.1. If

$$
\iint_{U_{0}} \frac{|\kappa|^{2}+\left|\mathscr{R} e^{-2 \imath \phi} \kappa\right|}{1-|\kappa|^{2}} \frac{d A}{|z|^{2}}<\infty
$$

and if

$$
\lim _{r \rightarrow 0} \arg w\left(r e^{i \theta_{0}}\right)=a
$$

for a particular value $\theta_{0}$ then $w(z)$ is conformal at $z=0$, i.e.,

$$
\lim _{z \rightarrow 0}(w(z) / z)=C, \quad C \neq 0 .
$$

The first significant results in this direction were given by Teichmüller [8] who proved that the condition

$$
\iint_{U_{0}} \frac{|\kappa|}{1-|\kappa|} \frac{d A}{|z|^{2}}<\infty
$$

implies that $|w(z)| \sim A|z|, A>0, z \rightarrow 0$ under the assumption that $w(z)$ is differentiable but not necessarily $K$ q.c. He conjectured that this condition was also sufficient to prove conformality at $z=0$ as in the following result.

THEOREM 1.2. If

$$
\iint_{U_{0}} \frac{|\kappa|}{1-|\kappa|} \frac{d A}{|z|^{2}}<\infty
$$

then $w(z)$ is conformal at $z=0$, i.e.,

$$
\lim _{z \rightarrow 0}(w(z) / z)=C, \quad C \neq 0 .
$$

The first proof of this result was presented by Belinskii $[1,2]$. It is usually referred to as the Teichmüller-Wittich-Belinskii theorem. Lehto [4] gave another derivation of this result as a consequence of some more general considerations and in [5] a modified exposition focused more on this particular problem clarifies some obscurities in Belinskii's work. However, all of the latter development uses strongly the assumption that the mapping is $K$ q.c. 
2. We will utilize the following two variants of [3; Lemma 3$]$.

LEMMA 2.1. Let $\gamma_{\jmath}, j=0,1, \cdots, n$, be a set of (closed) Jordan curves separ. ating 0 from $\infty$. Let

$$
\xi_{1}^{(j)}=\frac{1}{2 \pi} \min _{z \in \gamma_{j}} \log |z|^{-1}, \quad \xi_{2}^{(j)}=\frac{1}{2 \pi} \max _{z \in \gamma_{j}} \log |z|^{-1}
$$

with $\xi_{2}^{(j-1)}<\xi_{1}^{(j)}, j=1, \cdots, n$. Let $M$, be the module of the ring domain with boundary components $\gamma_{j-1}$ and $\gamma_{j}, j=1,2, \cdots, n$. Then

$$
\sum_{j=1}^{n} M_{\jmath} \leqq \xi_{1}^{(n)}-\xi_{2}^{(0)}+2-\sum_{j=1}^{n-1} f\left(\xi_{2}^{(j)}-\xi_{1}^{(j)}\right)
$$

where $f$ is a monotone increasing function independent of any geometric properties of the configuration.

The proof is a slight modification of that of $[3$; Lemma 3$]$. We set

$$
\begin{aligned}
& \rho(z)=0, \quad \frac{1}{2 \pi} \log |z|^{-1}<\xi_{2}^{(0)}-1, \quad \frac{1}{2 \pi} \log |z|^{-1}>\xi_{1}^{(n)}+1, \\
& \rho(z)=\left(1+\frac{1}{3}\left(\xi_{2}^{(j)}-\xi_{1}^{(j)}\right)^{2}\right)^{-1 / 2}, \quad \frac{1}{3}\left(2 \xi_{1}^{(j)}+\xi_{2}^{(j)}\right)<\frac{1}{2 \pi} \log |z|^{-1}<\frac{1}{3}\left(\xi_{1}^{(j)}+2 \xi_{2}^{(j)}\right), \\
& \rho=1, \quad \text { elsewhere. }
\end{aligned}
$$

It is readily verified that $\rho(z)(2 \pi|z|)^{-1}|d z|$ is an admissible metric for the module problems defining $M_{\jmath}, j=1, \cdots, n$. Therefore

$$
\begin{aligned}
\sum_{j=1}^{n} M_{\jmath} \leqq & \frac{1}{4 \pi^{2}} \iint \rho^{2}(z)|z|^{-2} d A_{2}=\xi_{1}^{(n)}-\xi_{2}^{(0)}+2 \\
& -\sum_{j=1}^{n-1} \frac{1}{9}\left(\xi_{2}^{(j)}-\xi_{1}^{(j)}\right)^{s}\left(1+\frac{1}{3}\left(\xi_{2}^{(j)}-\xi_{1}^{(j)}\right)^{2}\right)^{-1} .
\end{aligned}
$$

Setting $f(t)=(1 / 9) t^{3}\left(1+(1 / 3) t^{2}\right)^{-1}$ we have the result of the lemma.

LEMMA 2.2. Let $B=\left\{z: r_{1}<|z|<r_{2}\right\}, 0<r_{1}<r_{2}$. Let $\gamma_{1}, \gamma_{2}$ be non-intersecting Jordan arcs in $B$ joining its boundary components. Let $Q_{1}, Q_{2}$ be the two quadrangles with $\gamma_{1}, \gamma_{2}$ as one pair of opposite sides, the others being on $|z|=r_{1}$, $|z|=r_{2}$. Let $\delta$ be the angular oscillation of $\gamma_{1}$,

$$
\delta=\max _{z \in r_{1}} \arg z-\min _{z \in \gamma_{1}} \arg z
$$

for an assigned branch of the argument. Let $M\left(Q_{1}\right), M\left(Q_{2}\right)$ be the modules of $Q_{1}, Q_{2}$ for curves joining the sides on $|z|=r_{1},|z|=r_{2}$. Then

$$
M\left(Q_{1}\right)+M\left(Q_{2}\right) \leqq 2 \pi\left(\log \frac{r_{2}}{r_{1}}\right)^{-1}-g\left(\left(\log \frac{r_{2}}{r_{1}}\right)^{-1} \delta\right)
$$


where $g$ is a positive non-decreasing function independent of any geometric properties of the configuration.

We map $B$ slit along $\gamma_{2}$ by $w=\phi(z)=i\left(\log r_{2} / r_{1}\right)^{-1} \log z$. Let $\Gamma$ be the image of $\gamma_{1}, \Gamma_{1}$ and $\Gamma_{2}$ the images of $\gamma_{2}$ where $\Gamma_{2}$ is obtained from $\Gamma_{1}$ by a horizontal translation of amount $2 \pi\left(\log r_{2} / r_{1}\right)^{-1}$. Let $Q$ be the quadrangle with $\Gamma_{1}, \Gamma_{2}$ as one pair of opposite sides, the others being on $v=0, v=1(w=u+i v)$. Let

$$
\xi_{1}=\min _{w \in \Gamma} u, \quad \xi_{2}=\max _{w \in \Gamma} u .
$$

Let $\eta=\min \left(\xi_{2}-\xi_{1}, 2 \pi\right)$ and set

$$
\rho(z)=\left(1+\frac{1}{3} \eta^{2}\right)^{-1 / 2} \quad \text { in } \quad-\frac{1}{6} \eta<u-\frac{1}{2}\left(\xi_{1}+\xi_{2}\right)<\frac{1}{6} \eta
$$

and in all vertical strips obtained from this by horizontal translations of $2 \pi n\left(\log r_{2} / r_{1}\right)^{-1}, n$ integer,

$$
\rho(z)=1 \quad \text { elsewhere. }
$$

It is readily verified that $\rho(z)|d z|$ provides an admissible metric for the module problems corresponding to $M\left(Q_{1}\right), M\left(Q_{2}\right)$ under the mapping $\phi$. Thus

$$
M\left(Q_{1}\right)+M\left(Q_{2}\right) \leqq \iint_{Q} \rho^{2} d A=2 \pi\left(\log \frac{r_{2}}{r_{1}}\right)^{-1}-\frac{1}{9} \eta^{3}\left(1+\frac{1}{3} \eta^{2}\right)^{-1}
$$

and if we set $g(t)=f(t), t \leqq 2 \pi, g(t)=f(2 \pi), t \geqq 2 \pi$ we have the result of the lemma.

3. Let $w(z)$ be the mapping introduced in $\S 1$. Let $0<r_{1}<r_{2}<1, B\left(r_{1}, r_{2}\right)=$ $\left\{z: r_{1}<|z|<r_{2}\right\}$ and let $B^{*}\left(r_{1}, r_{2}\right)$ be its image under $w(z)$. We denote by $M\left(r_{1}, r_{2}\right)$ the module of the family of curves separating the contours of $B\left(r_{1}, r_{2}\right)$ so that

$$
M\left(r_{1}, r_{2}\right)=\frac{1}{2 \pi} \log \frac{r_{2}}{r_{1}}
$$

and by $M^{*}\left(r_{1}, r_{2}\right)$ the corresponding module for $B^{*}\left(r_{1}, r_{2}\right)$.

Let $z=r e^{\imath \phi}$ be a regular point for $w(z), \kappa(z)$ the complex dilatation at this point so that the expressions

$$
h_{1}(z)=\frac{\left|1+e^{-2 \imath \phi} \kappa\right|^{2}}{1-|\kappa|^{2}}, \quad h_{2}(z)=\frac{\left|1-e^{-2 i \phi} \kappa\right|^{2}}{1-|\kappa|^{2}}
$$

are defined a. e. in $U_{0}$. dition.

From this point on we will assume that $w(z)$ satisfies the following con- 
CONDITION 3.1.

$$
h_{1}^{*}(\phi)=\int_{r_{1}}^{r_{2}} h_{1}\left(r e^{2 \phi}\right) \frac{d r}{r}, \quad h_{2}^{*}(r)=\int_{0}^{2 \pi} h_{2}\left(r e^{i \phi}\right) d \phi
$$

exist a. e. for $\phi \in[0,2 \pi], r \in\left[r_{1}, r_{2}\right], 0<r_{1}<r_{2}<1$ and further $h_{1}^{*}(\phi) \in L^{1}[0,2 \pi]$, $h_{2}^{*}(r) \in L^{1}\left[r_{1}, r_{2}\right]$.

As in [6] it is easily shown that

$$
\begin{aligned}
& M *\left(r_{1}, r_{2}\right) \leqq\left[\int_{0}^{2 \pi}\left(\int_{r_{1}}^{r_{2}} h_{1}\left(r e^{i \phi}\right) \frac{d r}{r}\right)^{-1} d \phi\right]^{-1} \\
& M *\left(r_{1}, r_{2}\right) \geqq \int_{r_{1}}^{r_{2}}\left(\int_{0}^{2 \pi} h_{2}\left(r e^{2 \phi}\right) d \phi\right)^{-1} \frac{d r}{r} .
\end{aligned}
$$

These lead to the proof of the following lemma.

LEMMA 3.1. We have

$$
\left|M *\left(r_{1}, r_{2}\right)-\frac{1}{2 \pi} \log \frac{r_{2}}{r_{1}}\right| \leqq \frac{1}{2 \pi^{2}} \iint_{B\left(r_{1}, r_{2}\right)} \frac{|\kappa|^{2}+\left|\mathcal{R} e^{-22 \phi} \kappa\right|}{1-|\kappa|^{2}} \frac{d A_{z}}{|z|^{2}} .
$$

The Cauchy-Schwarz inequality implies that

$$
M *\left(r_{1}, r_{2}\right) \leqq \frac{1}{4 \pi^{2}} \int_{0}^{2 \pi} h_{1}^{*}(\phi) d \phi
$$

and

$$
\left(M *\left(r_{1}, r_{2}\right)\right)^{-1} \leqq\left(\log \frac{r_{2}}{r_{1}}\right)^{-2} \int_{r_{1}}^{r_{2}} h_{2}^{*}(r) \frac{d r}{r} .
$$

Therefore for $M *\left(r_{1}, r_{2}\right)<M\left(r_{1}, r_{2}\right)$

$$
\begin{aligned}
M *\left(r_{1}, r_{2}\right)-M\left(r_{1}, r_{2}\right) & \leqq \frac{1}{4 \pi^{2}} \frac{M *\left(r_{1}, r_{2}\right)}{M\left(r_{1}, r_{2}\right)} \iint_{B\left(r_{1}, r_{2}\right)}\left|h_{2}-1\right| \frac{d A_{z}}{|z|^{2}} \\
& \leqq \frac{1}{4 \pi^{2}} \iint_{B\left(r_{1}, r_{2}\right)}\left|h_{2}-1\right| \frac{d A_{2}}{|z|^{2}}
\end{aligned}
$$

while for $M\left(r_{1}, r_{2}\right) \leqq M *\left(r_{1}, r_{2}\right)$

$$
M *\left(r_{1}, r_{2}\right)-M\left(r_{1}, r_{2}\right) \leqq \frac{1}{4 \pi^{2}} \iint_{B\left(r_{1}, r_{2}\right)}\left|h_{1}-1\right| \frac{d A_{z}}{|z|^{2}} .
$$

It is seen at once that

$$
\begin{aligned}
& h_{1}(z)-1=2 \frac{|\kappa|^{2}+\mathscr{R} e^{-22 \phi} \kappa}{1-|\kappa|^{2}} \\
& h_{2}(z)-1=2 \frac{|\kappa|^{2}-\mathcal{R} e^{-22 \phi} \kappa}{1-|\kappa|^{2}}
\end{aligned}
$$

from which (3.2) follows.

Let $Q$ denote the quadrangle obtained from the domain $\left\{r_{1}<|z|<r_{2}, \theta_{1}<\right.$ 
$\left.\arg z<\theta_{2}\right\}$ by assigning as opposite pairs of sides the boundary sets on radii and circles. Let $M(Q)$ be the module of $Q$ for curves joining the latter sides. Let $\hat{Q}$ be the image of $Q$ under $w(z)$ and $M(\hat{Q})$ its corresponding module.

LEMMA 3.2. We have

$$
M(Q)-M(\hat{Q}) \leqq 2\left(\log \frac{r_{2}}{r_{1}}\right)^{-2} \int_{\theta_{1}}^{\theta_{2}} \int_{r_{1}}^{r_{2}} \frac{|\kappa|^{2}+\left|\mathcal{R} e^{-2 \imath \hat{\phi}} \kappa\right|}{1-|\kappa|^{2}} \frac{d A_{z}}{|z|^{2}} .
$$

As before

$$
M(\hat{Q}) \geqq \int_{\theta_{1}}^{\theta_{2}}\left(\int_{r_{1}}^{r_{2}} h_{1}\left(r e^{\imath \phi}\right) \frac{d r}{r}\right)^{-1} d \phi .
$$

Applying the Cauchy-Schwarz inequality we get

$$
M(\hat{Q})^{-1} \leqq\left(\theta_{2}-\theta_{1}\right)^{-2} \int_{\theta_{1}}^{\theta_{2}} \int_{r_{1}}^{r_{2}} h_{1}\left(r e^{2 \phi}\right) \frac{d r}{r} d \phi .
$$

Since evidently $M(Q)=\left(\log r_{2} / r_{1}\right)^{-1}\left(\theta_{2}-\theta_{1}\right)$ we find that if $M(\hat{Q})<M(Q)$

$$
M(Q)-M(\hat{Q}) \leqq \frac{M(\hat{Q})}{M(Q)}\left(\log \frac{r_{2}}{r_{1}}\right)^{-2} \int_{\theta_{1}}^{\theta_{2}} \int_{r_{1}}^{r_{2}}\left|h_{1}\left(r e^{\imath \phi}\right)-1\right| \frac{d A_{z}}{|z|^{2}}
$$

from which (3.3) follows. The inequality is trivial in the alternative case.

4. LEMMA 4.1. For the mapping $w(z)$ let

$$
m_{2}(r)=\max _{|\boldsymbol{z}|=r}|w(z)|, \quad m_{1}(r)=\min _{|z|=r}|w(z)| .
$$

Then if $w(z)$ satisfies Condition 3.1 and

$$
\iint_{U_{0}} \frac{|\kappa|^{2}+\left|\mathscr{R} e^{-2 i \phi} \kappa\right|}{1-|\kappa|^{2}} \frac{d A_{z}}{|z|^{2}}<\infty
$$

we have $\lim _{r \rightarrow 0} m_{2}(r) / m_{1}(r)=1$.

In the contrary case there would exist $\lambda>0$ and a sequence of values $\left\{r_{n}\right\}$, $r_{n}<1, r_{n} \downarrow 0$ such that $m_{1}\left(r_{n}\right)>m_{2}\left(r_{n+1}\right)$ and

$$
\log \frac{m_{2}\left(r_{n}\right)}{m_{1}\left(r_{n}\right)}>\lambda, \quad n=1,2, \cdots .
$$

For arbitrary $\varepsilon>0$ assume that $r_{1}$ is sufficiently small that

$$
\frac{1}{2 \pi^{2}} \iint_{B\left(r_{m}, r_{n}\right)} \frac{|\kappa|^{2}+\left|\mathcal{R} e^{-2 \imath \phi} \kappa\right|}{1-|\kappa|^{2}} \frac{d A_{z}}{|z|^{2}}<\varepsilon
$$

for $m>n>1$. Using the monotonicity property of the module and Lemma 3.1 we conclude that for any integer $N>1$

$$
\frac{1}{2 \pi} \log \frac{m_{1}\left(r_{1}\right)}{m_{2}\left(r_{N+1}\right)} \leqq M *\left(r_{N+1}, r_{1}\right) \leqq \frac{1}{2 \pi} \log \frac{r_{1}}{r_{N+1}}+\varepsilon
$$


and

$$
\frac{1}{2 \pi} \log \frac{r_{1}}{r_{N+1}}-N \varepsilon \leqq \sum_{j=1}^{N} M *\left(r_{j+1}, r_{\jmath}\right) .
$$

Applying Lemma 2.1 to the right-hand side of inequality (4.4) we have

$$
\sum_{j=1}^{N} M *\left(r_{j+1}, r_{j}\right) \leqq \frac{1}{2 \pi} \log \frac{m_{1}\left(r_{1}\right)}{m_{2}\left(r_{N+1}\right)}+2-\sum_{j=2}^{N} f\left(\log \frac{m_{2}\left(r_{j}\right)}{m_{1}\left(r_{j}\right)}\right) .
$$

Since by (4.2) $f\left(\log m_{2}\left(r_{\jmath}\right) / m_{1}\left(r_{\jmath}\right)\right) \geqq f(\lambda)$ from (4.3), (4.4) and (4.5) follows

and

$$
\frac{1}{2 \pi} \log \frac{r_{1}}{r_{N+1}}-(N+1) \varepsilon \leqq \frac{1}{2 \pi} \log \frac{r_{1}}{r_{N+1}}+2-(N-1) f(\lambda)
$$

$$
(N-1) f(\lambda) \leqq 2+(N+1) \varepsilon .
$$

Dividing by $N$ and letting $N$ tend to infinity we obtain the contradiction $f(\lambda) \leqq \varepsilon$.

LEMMA 4.2. Under the conditions of Lemma 4.1 there exists a constant $C>0$ such that $|w(z)| \sim C|z|$ as $|z|$ tends to 0 .

This is shown by proving that

$$
\lim _{r \rightarrow 0} \log \frac{r}{m_{2}(r)}=C, \quad C \neq 0 .
$$

Given $\varepsilon>0$ it follows from the monotonicity property of the module and Lemma 4.1 that there exists $\delta=\delta(\varepsilon)$ such that for $0<r_{1}<r_{2}<\delta$.

$$
\left|M *\left(r_{1}, r_{2}\right)-\frac{1}{2 \pi} \log \frac{m_{2}\left(r_{2}\right)}{m_{2}\left(r_{1}\right)}\right|<\varepsilon
$$

while by Lemma 3.1

$$
\left|M^{*}\left(r_{1}, r_{2}\right)-\frac{1}{2 \pi} \log \frac{r_{2}}{r_{1}}\right|<\varepsilon .
$$

From these and the Cauchy criterion (4.6) follows.

5. For $0<r_{1}<r_{2}, \theta_{1}<\theta_{2}<\theta_{1}+2 \pi$ the set of points $r e^{i \theta}: r_{1}<r<r_{2}, \theta_{1}<\theta<\theta_{2}$ becomes a quadrangle on assigning the boundary arcs on $r=r_{1}, r=r_{2}$ as a pair of opposite sides. It is denoted by $Q\left(r_{1}, r_{2} ; \theta_{1}, \theta_{2}\right)$. Its module for the curves join'ng this pair of opposite sides is $\left(\theta_{2}-\theta_{1}\right)\left(\log r_{2} / r_{1}\right)^{-1}$.

LEMMA 5.1. If $w(z)$ is a homeomorphism of a punctured neighborhood of $z=0$ onto a punctured neighborhood of $w=0$ with the origins corresponding as boundary components and satisfies the conditions

(a) $|w(z)| \sim A|z|, A>0$, as $z \rightarrow 0$, 
(b) given $\varepsilon>0$ there exists $R=R(\varepsilon)$ such that for $r_{2}<R$ and for $\hat{Q}$ the quadrangle image of $Q\left(r_{1}, r_{2} ; \theta_{1}, \theta_{2}\right)$ under $w(z)$ with module $m(\hat{Q})$ corresponding to that above

$$
m(\hat{Q})>\left(\theta_{2}-\theta_{1}\right)\left(\log \frac{r_{2}}{r_{1}}\right)^{-1}-\varepsilon,
$$

then $w(z)$ is asymptotically a rotation, i.e., the expression (1.3) trends to zero uniformly in $\theta_{1}$ and $\theta_{2}$ as $r$ tends to zero.

If this were not the case there would exist $\delta>0$ and a sequence of values of $r \downarrow 0$ such that for a suitable choice of notation

$$
\phi_{2}-\phi_{1} \leqq \theta_{2}-\theta_{1}-\delta
$$

where $\phi_{2}=\arg w\left(r e^{i \theta_{2}}\right), \phi_{1}=\arg w\left(r e^{i \theta_{1}}\right)$. For such values of $r$ we choose $r_{2}=2 r$, $r_{1}=(1 / 2) r$ and denote

$$
r_{2}^{*}=\min _{|z|=r_{2}}|w(z)|, \quad r_{1}^{*}=\max _{|z|=r_{1}}|w(z)| .
$$

For $r$ sufficiently small the image of $|z|=r$ under $w=w(z)$ will lie in the ring $r_{1}^{*}<|w|<r_{2}^{*}$. Let $\hat{Q}_{1}$ be the quadrangle image of $Q\left(r_{1}, r_{2} ; \theta_{1}, \theta_{2}\right), \hat{Q}_{2}$ the quadrangle image of $Q\left(r_{1}, r_{2} ; \theta_{2}, \theta_{1}+2 \pi\right), \gamma_{1}$ the image of the segment $\left\{\rho e^{i \theta} ; \theta=\theta_{1}\right.$, $\left.r_{1}<\rho<r_{2}\right\}, \gamma_{2}$ the image of the segment $\left\{\rho e^{i \theta}: \theta=\theta_{2}, r_{1}<\rho<r_{2}\right\}$. Follow $\gamma_{3}$ from $w\left(r e^{i \theta j}\right)$ in each sense until we meet respectively $|w|=r_{1}^{*},|w|=r_{2}^{*}$, obtaining an $\operatorname{arc} \gamma_{3}^{*}, j=1,2$. The arcs $\gamma_{1}^{*}, \gamma_{2}^{*}$ together with arcs on $|w|=r_{1}^{*},|w|=r_{2}^{*}$ determine quadrangles $Q_{1}^{*}, Q_{2}^{*}$ with modules $m\left(Q_{1}^{*}\right), m\left(Q_{2}^{*}\right)$ chosen as above such that $m\left(Q_{1}^{*}\right) \geqq m\left(\hat{Q}_{1}\right), m\left(Q_{2}^{*}\right) \geqq m\left(\hat{Q}_{2}\right)$. We consider two cases depending on the angular oscillation of $\gamma_{1}^{*}, \gamma_{2}^{*}$. Then

CASE A. The angular oscillation on each of $\gamma_{1}^{*}$ and $\gamma_{2}^{*}$ is less than $(1 / 4) \delta$.

$$
m\left(\hat{Q}_{1}\right) \leqq m\left(Q_{1}^{*}\right) \leqq\left(\log \frac{r_{2}^{*}}{r_{1}^{*}}\right)^{-1}\left(\phi_{2}-\phi_{1}+\frac{1}{2} \delta\right)
$$

By condition (b)

$$
\begin{aligned}
m\left(\hat{Q}_{1}\right) & >(\log 4)^{-1}\left(\theta_{2}-\theta_{1}\right)-\varepsilon \\
& >(\log 4)^{-1}\left(\phi_{2}-\phi_{1}+\delta\right)-\varepsilon .
\end{aligned}
$$

Since by condition (a) as $r$ tends to $0, \log r_{2}^{*} / r_{1}^{*}$ tends to $\log 4$ for $\varepsilon$ sufficiently small this provides a contradiction.

CASE B. The angular oscillation on one of $\gamma_{1}^{*}, \gamma_{2}^{*}$ is at least $(1 / 4) \delta$. Then by Lemma 2.2 


$$
\begin{aligned}
m\left(\hat{Q}_{1}\right)+m\left(\hat{Q}_{2}\right) & \leqq m\left(Q_{1}^{*}\right)+m\left(Q_{2}^{*}\right) \\
& \leqq 2 \pi\left(\log \frac{r_{2}^{*}}{r_{1}^{*}}\right)^{-1}-g\left(\frac{1}{4} \delta\left(\log \frac{r_{2}^{*}}{r_{1}^{*}}\right)^{-1}\right) .
\end{aligned}
$$

On the other hand by condition (b)

$$
m\left(\hat{Q}_{1}\right)+m\left(\hat{Q}_{2}\right)>2 \pi(\log 4)^{-1}-2 \varepsilon .
$$

Since by condition (a) as $r$ tends to $0, \log r_{2}^{*} / r_{1}^{*}$ tends to $\log 4$ for $\varepsilon$ sufficiently small this provides a contradiction.

COROLlaRRY 5.1. If $w(z)$ satisfies Condition 3.1 and (4.1) it is asymptotically a rotation on circles as $z$ tends to 0 .

Condition (a) follows from Lemma 4.2, condition (b) from Lemma 3.2.

6. Let $\hat{U}_{0}$ be the unit disc $U_{0}$ slit along the radius $\{(x, y): 0 \leqq x<1, y=0\}$. We map $\hat{U}_{0}$ and its image $w\left(\hat{U}_{0}\right)$ onto semistrips $S_{1}$ and $S_{2}$ using in each case a branch of $-\log$. Let $f(\sigma)=-\log \left(w\left(e^{-\sigma}\right)\right)$ be the map from $S_{1}$ to $S_{2}$ induced by the mapping $w(z), f(\sigma)$ is extended throughout the half-plane $\mathscr{R} \sigma>0$ as a continuous function by setting

$$
f(\sigma+2 k \pi i)=f(\sigma)+2 k \pi i
$$

for every integer $k$. Setting $\sigma=s+i t, f(\sigma)=u(s, t)+i v(s, t)$ is an ACL homeomorphism and $\lim _{s \rightarrow \infty} u(s, t)=+\infty$.

LEMMA 6.1. If

$$
\lim _{s \rightarrow \infty}(u(s, t)-s)=A
$$

for $A$ finite uniformly in $t$,

$$
\lim _{s \rightarrow \infty}\left(v\left(s, t_{2}\right)-v\left(s, t_{1}\right)-\left(t_{2}-t_{1}\right)\right)=0
$$

uniformly in $t_{1}$ and $t_{2}$ and

$$
\int_{0}^{\infty} \int_{0}^{2 \pi}\left|\frac{1}{2} \frac{\left(u_{s}+u_{t}\right)^{2}+\left(v_{s}+v_{t}\right)^{2}}{u_{s} v_{t}-u_{t} v_{s}}-1\right| d s d t<\infty
$$

then there exists a finite value a such that

$$
\lim _{s \rightarrow \infty}(v(s, t)-t)=a
$$

uniformly in $t$.

We assume that (6.4) does not hold for a certain $t$. Then there exist sequences $\left\{s_{n}^{(1)}\right\}$ and $\left\{s_{n}^{(2)}\right\}, n=1,2, \cdots$, tending to $\infty, s_{n}^{(1)}<s_{n}^{(2)}$, such that 


$$
\delta(t)=\lim _{n \rightarrow \infty}\left(v\left(s_{n}^{(2)}, t\right)-v\left(s_{n}^{(1)}, t\right)\right) \neq 0 .
$$

Because of (6.2), $\delta(t)=\delta$, independent of $t$. We may assume $\delta>0$, the case where $\delta<0$ is handled analogously. Let $\varepsilon>0$ be a fixed small number and let $N=N(\varepsilon)$ be big enough so that for $n>N$

$$
\left|u\left(s_{n}^{(i)}, t\right)-s_{n}^{(i)}-A\right|<\varepsilon, \quad i=1,2,
$$

for any $t$,

$$
\left|v\left(s_{n}^{(i)}, t_{2}\right)-v\left(s_{n}^{(i)}, t_{1}\right)-\left(t_{2}-t_{1}\right)\right|<\varepsilon, \quad i=1,2,
$$

for any $t_{1}, t_{2}$,

$$
v\left(s_{n}^{(2)}, t\right)-v\left(s_{n}^{(1)}, t\right)-\delta>-\varepsilon
$$

for any $t$ and

$$
\int_{s_{n}^{(1)}}^{s_{n}^{(2)}} \int_{0}^{2 \pi}\left|\frac{1}{2} \frac{\left(u_{s}+u_{t}\right)^{2}+\left(v_{s}+v_{t}\right)^{2}}{u_{s} v_{t}-u_{t} v_{s}}-1\right| d A_{\sigma}<\varepsilon .
$$

Let $s_{1}$ and $s_{2}$ stand for $s_{n}^{(1)}$ and $s_{n}^{(2)}$ for a fixed $n>N$. Consider the map

$$
\boldsymbol{\sigma}=g(p, q)=(p, p+q) \text {. }
$$

It maps the rectangle $Q=\left\{(p, q): s_{1}<p<s_{2}, 0<q<2 \pi\right\}$ onto a parallelogram $P$ in the $\sigma$-plane. Denote by $P^{*}$ the image of $P$ under $f$ and let $h=f \circ g$.

We study the module $M\left(\Gamma^{*}\right)$ of the family of curves $\Gamma^{*}=\left\{\gamma_{q}^{*}\right\}_{0<q<2 \pi}$, where $\gamma_{q}^{*}$ is the image under $h$ of the horizontal segment $I_{q}=\left\{(p, q): s_{1}<p<s_{2}, q\right.$ fixed, $0<q<2 \pi\}$. By [7; Theorem 14] we have

$$
M\left(\Gamma^{*}\right)=\int_{0}^{2 \pi}\left[\int_{s_{1}}^{s_{2}} \frac{|d h / d p|^{2}}{J_{h}} d p\right]^{-1} d q,
$$

where $J_{h}$ denotes the Jacobian of the map $h$. Since

$$
\iint_{Q} \frac{|d h / d p|^{2}}{J_{h}} d p d q=\iint_{P} \frac{\left(u_{s}+u_{t}\right)^{2}+\left(v_{s}+v_{t}\right)^{2}}{u_{s} v_{t}-u_{t} v_{s}} d A_{o}
$$

and by the Cauchy-Schwarz inequality

$$
M\left(\Gamma^{*}\right) \geqq(2 \pi)^{2}\left[\iint_{Q} \frac{|d h / d p|^{2}}{J_{h}} d p d q\right]^{-1}
$$

it follows from (6.8) that

$$
M\left(\Gamma^{*}\right) \geqq(2 \pi)^{2}\left[4 \pi\left(s_{2}-s_{1}\right)+2 \varepsilon\right]^{-1} .
$$

An estimate for $M\left(\Gamma^{*}\right)$ from above can be obtained using the definition of the module of a curve family. Using (6.5), (6.6), (6.7) we conclude that

$$
\text { area } P^{*} \leqq 2 \pi\left(\left(s_{2}-s_{1}\right)+2 \varepsilon\right)
$$




$$
\text { (length } \left.\gamma_{q}^{*}\right)^{2} \geqq\left(\left(s_{2}-s_{1}\right)-2 \varepsilon\right)^{2}+\left(\left(s_{2}-s_{1}\right)-3 \varepsilon+\delta\right)^{2}, \quad 0<q<2 \pi
$$

so that

$$
M\left(\Gamma^{*}\right) \leqq \frac{2 \pi\left(\left(s_{2}-s_{1}\right)+2 \varepsilon\right)}{\left(\left(s_{2}-s_{1}\right)-2 \varepsilon\right)^{2}+\left(\left(s_{2}-s_{1}\right)-3 \varepsilon+\delta\right)^{2}} .
$$

Since

$$
\left(\left(s_{2}-s_{1}\right)-2 \varepsilon\right)^{2}+\left(\left(s_{2}-s_{1}\right)+\delta-3 \varepsilon\right)^{2} \geqq 2\left(s_{2}-s_{1}\right)^{2}+2\left(s_{2}-s_{1}\right)(\delta-5 \varepsilon)+\frac{2}{\pi} \varepsilon^{2}
$$

comparing (6.9) and (6.10) we obtain

$$
2 \pi\left(s_{2}-s_{1}\right)^{2}+2 \pi\left(s_{2}-s_{1}\right)(\delta-5 \varepsilon)+2 \varepsilon^{2} \leqq 2 \pi\left(s_{2}-s_{1}\right)^{2}+(4 \pi+1) \varepsilon\left(s_{2}-s_{1}\right)+2 \varepsilon^{2}
$$

and

$$
2 \pi(\delta-5 \varepsilon) \leqq(4 \pi+1) \varepsilon
$$

which for $\varepsilon$ sufficiently small provides a contradiction.

The proof of Theorem 1.2 is now immediate. The hypothesis there implies (1.1) so that (1.2) and (1.3) hold. Transforming to the logarithmic plane as above we obtain (6.1) and (6.2). If $\kappa_{0}$ is the complex dilatation $f_{\bar{\sigma}} / f_{\sigma}$, we obtain

$$
\iint_{S_{1}} \frac{\left|\kappa_{0}\right|}{1-\left|\kappa_{0}\right|^{2}} d A_{\sigma}<\infty
$$

Since

$$
\frac{1}{2} \frac{\left(u_{s}+u_{t}\right)^{2}+\left(v_{s}+v_{t}\right)^{2}}{u_{s} v_{t}-u_{t} v_{s}}-1=\frac{\left|1+i \kappa_{0}\right|^{2}}{1-\left|\kappa_{0}\right|^{2}}-1=\frac{2\left|\kappa_{0}\right|^{2}+2 \mathscr{R} i \kappa_{0}}{1-\left|\kappa_{0}\right|^{2}}
$$

it is clear that (6.11) implies (6.3) so that by Lemma 6.1

$$
\lim _{r \rightarrow \infty}\left(\arg w\left(r e^{i \theta}\right)-\theta\right)=a
$$

uniformly with respect to $\theta$ and finally

$$
\lim _{|z| \rightarrow 0}(\arg w(z)-\arg z)=-a
$$

completing the proof.

7. Let $S$ be the strip in the $\sigma$-plane, $\sigma=s+i t$, defined by

$$
S=\{\sigma: s \geqq 1,0 \leqq t \leqq 2 \pi\}
$$

and let $f(\sigma)$ be an ACL homeomorphism of $S$ onto itself with $1,1+2 \pi i$ and $\infty$ as fixed points. We denote by $\kappa_{0}$ the complex dilatation $f_{\bar{\sigma}} / f_{\sigma}$. We assume that for $1<s_{1}<s_{2}<\infty$ the following integrals exist

$$
\iint_{\left(s_{1}<\Omega \sigma<s_{2}\right) \cap S} \frac{\left|1+\kappa_{0}\right|^{2}}{1-\left|\kappa_{0}\right|^{2}} d A_{\sigma}, \quad \iint_{\left(s_{1}<R \sigma<s_{2}\right) \cap S} \frac{\left|1-\kappa_{0}\right|^{2}}{1-\left|\kappa_{0}\right|^{2}} d A_{\sigma} .
$$

THEOREM 7.1. If 


$$
\iint_{S} \frac{\left|\kappa_{0}\right|^{2}+\left|R \kappa_{0}\right|}{1-\left|\kappa_{0}\right|^{2}} d A_{\sigma}<\infty
$$

then

$$
f(\sigma)=A+s+i t+o(1)
$$

as $s \rightarrow \infty$ uniformly in $t$.

We may assume that $f(s+2 \pi i)=f(s), s$ real, since this can be attained by reflecting the strip in a horizontal side and adjusting the dimensions without affecting the conditions on $f$, in particular (7.1). Then $w(z)=\exp \left(-f\left(\log z^{-1}\right)\right)$ is a homeomorphism from $U_{0}$ onto itself with $w(0)=0$ and complex dilatation $\kappa$. From (7.1) follows

$$
\iint_{U_{0}} \frac{|\kappa|^{2}+\mathscr{R}\left(e^{-2 i \theta} \kappa\right)}{1-|\kappa|^{2}} \frac{d A_{z}}{|z|^{2}}<\infty .
$$

Since $w(z)$ maps the radius $\{(x, y): 0 \leqq x<1, y=0\}$ onto itself from Corollary 1.1 follows that $w(z) \sim C z$ as $z$ tends to $0, C \neq 0$, which implies (7.2).

Theorem 7.1 is a stronger result than the following strip variant of the Teichmüller-Wittich-Belinski theorem.

THEOREM 7.2. If $f$ satisfies the condition

$$
\iint_{S} \frac{\left|\kappa_{0}\right|}{1-\left|\kappa_{0}\right|} d A_{\sigma}<\infty
$$

then

$$
f(\sigma)=A+s+i t+o(1)
$$

as $s \rightarrow \infty$ uniformly in $t$.

Indeed (7.3) implies (7.2) but the mapping defined by $f=h \circ g$ where $g(\sigma)=$ $s+i(t+\log s)$ maps $S$ onto a strip $\hat{S}$ and $h$ maps $\hat{S}$ conformally onto $S$ has complex dilatation

$$
\kappa_{0}(\sigma)=\frac{1+2 i s}{4 s^{2}+1} .
$$

This satisfies condition (7.2) without satisfying (7.3).

\section{BIBLIOGRAPHY}

[1] P.P. BELINSKII, Behavior of a quasiconformal mapping at an isolated singular point, Uchenye Zapiski Lvovskii Gosudarsvennyi Universitet Ser. MekhanikoMatematichna, 29 (1954), pp. 58-70 (in Russian).

[2] P.P. BelinskiI, General Properties of Quasiconformal Mappings, Nauka, Novosibirsk, 1974 (in Russian). 
[3] James A. Jenkins, On the Phragmén-Lindelöf theorem, the Denjoy conjecture and related results, Mathematical Essays Dedicated to A.J. Macintyre, Ohio University Press, Athens, Ohio, 1970, pp. 183-200.

[4] O. LeHTO, On the differentiability of quasiconformal mappings with prescribed complex dilatation, Ann. Acad. Sci. Fenn. Ser. A 1 Math. 275 (1960), pp. 1-28.

[5] O. Lehto And K. Virtanen, Quasiconformal Mappings in the Plane, SpringerVerlag, Berlin-Heidelberg-New York, 1973.

[6] E. REICH AND H. WALCZAK, On the behavior of quasiconformal mappings at a point, Trans. Amer. Math. Soc., 117 (1965), pp. 335-351.

[7] B. Rodin, The method of extremal length, Bull. Amer. Math. Soc., 80 (1974), pp. 587-606.

[8] O. TeICHMüLLER, Untersuchungen über konforme und quasikonforme Abbildungen, Deutsche Mathematik, 3 (1938), pp. 621-678.

InSTITUE OF MATHEMATICS

P.O. Box 373

1090 Sofia, Bulgaria

Department of MATHEMATICS

WASHINGTON UNIVERSITY

CAMPuS Box 1146

One Brookings Drive

ST. Louis, Missouri 63130-4899

U.S.A. 